\title{
Spinocerebellar Ataxia Type 13 Mutant Potassium Channel Alters Neuronal Excitability and Causes Locomotor Deficits in Zebrafish
}

\author{
Fadi A. Issa, ${ }^{1}$ Christopher Mazzochi, ${ }^{1}$ Allan F. Mock, ${ }^{1}$ and Diane M. Papazian ${ }^{1,2,3}$ \\ ${ }^{1}$ Department of Physiology, ${ }^{2}$ Molecular Biology Institute, and ${ }^{3}$ Brain Research Institute, David Geffen School of Medicine, University of California at Los \\ Angeles, Los Angeles, California 90095-1751
}

\begin{abstract}
Whether changes in neuronal excitability can cause neurodegenerative disease in the absence of other factors such as protein aggregation is unknown. Mutations in the Kv3.3 voltage-gated $\mathrm{K}^{+}$channel cause spinocerebellar ataxia type 13 (SCA13), a human autosomaldominant disease characterized by locomotor impairment and the death of cerebellar neurons. Kv3.3 channels facilitate repetitive, high-frequency firing of action potentials, suggesting that pathogenesis in SCA13 is triggered by changes in electrical activity in neurons. To investigate whether SCA13 mutations alter excitability in vivo, we expressed the human dominant-negative R420H mutant subunit in zebrafish. The disease-causing mutation specifically suppressed the excitability of Kv3.3-expressing, fast-spiking motor neurons during evoked firing and fictive swimming and, in parallel, decreased the precision and amplitude of the startle response. The dominantnegative effect of the mutant subunit on $\mathrm{K}^{+}$current amplitude was directly responsible for the reduced excitability and locomotor phenotype. Our data provide strong evidence that changes in excitability initiate pathogenesis in SCA13 and establish zebrafish as an excellent model system for investigating how changes in neuronal activity impair locomotor control and cause cell death.
\end{abstract}

\section{Introduction}

The spinocerebellar ataxias (SCAs) are a group of human autosomal-dominant diseases characterized by motor impairment, oculomotor abnormalities, and the death of cerebellar neurons (Paulson, 2009). These rare monogenic disorders provide an opportunity to investigate pathogenic mechanisms that may contribute to the etiology of more common neurodegenerative diseases. Among SCA mutations that have been molecularly characterized, the majority are nucleotide repeat expansions, suggesting that protein aggregation is central to the development of disease in these cases.

Recently, KCNC3, which encodes the Kv3.3 voltage-gated $\mathrm{K}^{+}$ channel, was identified as the gene mutated in spinocerebellar ataxia type 13 (SCA13) (Waters et al., 2006). SCA13 is the first neurodegenerative disease known to be caused by $\mathrm{K}^{+}$channel mutations (Nelson, 2006). SCA13 exists in two forms characterized by infant or adult onset depending on the causative mutation, leading

\footnotetext{
Received Dec. 16, 2010; revised March 18, 2011; accepted March 21, 2011

Author contributions: D.M.P. designed research; F.A.I., C.M., A.F.M., and D.M.P. performed research; F.A.I., C.M., and D.M.P. analyzed data; F.A.I. and D.M.P. wrote the paper.

This work was supported by National Institutes of Health (NIH) Grant R01 NS058500 to D.M.P. F.A.I. and C.M. were partially supported by NIH Training Grant T32 NS007101. We are grateful to Drs. David Glanzman, Nancy Wayne, and members of the Papazian laboratory for comments on this manuscript. We thank Drs. Pierre Drapeau, Judith Eisen, Alexandra Tallafuss, and Harry Burgess for advice and assistance and Alvaro Sagasti for the loan of a Redlake MotionMeter high-speed video camera. Leena Patel provided excellent technical assistance. We gratefully acknowledge the help of Yuan Dong and Haigen Huang of the University of California at Los Angeles (UCLA) Zebrafish Core Facility and Matthew Schibler of the Carol Moss Spivak Cell Imaging Center (UCLA Brain Research Institute).

Correspondence should be addressed to Dr. Diane M. Papazian, Department of Physiology, David Geffen School of Medicine at UCLA, Los Angeles, CA 90095-1751. E-mail: papazian@mednet.ucla.edu.

DOI:10.1523/JNEUROSCI.6572-10.2011

Copyright $\odot 2011$ the authors $\quad 0270-6474 / 11 / 316831-11 \$ 15.00 / 0$
}

to severe maldevelopment or progressive degeneration of the cerebellum, respectively. Kv3.3 channels, like other Kv3 family members, have novel gating properties, including a depolarized activation range, fast activation, and very fast deactivation, that facilitate sustained, high-frequency firing in neurons (Rudy and McBain, 2001). Disease-causing mutations alter voltagedependent gating or reduce $\mathrm{K}^{+}$current amplitude, raising the possibility that the locomotor deficits and neuronal loss seen in SCA13 result from changes in the excitability of Kv3.3-expressing cells (Waters et al., 2006; Figueroa et al., 2010). Although changes in neuronal function have been reported in neurodegenerative diseases caused by abnormal protein accumulation, it has been difficult to determine whether altered activity contributes to pathogenesis (Supnet and Bezprozvanny, 2010). In contrast, SCA13 provides a novel opportunity to investigate the role of electrical excitability in motor control and neuronal survival in the absence of other complicating factors such as protein misfolding or aggregation.

To determine whether SCA13 mutations alter neuronal excitability in vivo, we expressed a dominant-negative SCA13 mutant subunit in the zebrafish, Danio rerio. We now report that the SCA13 mutation specifically suppresses the excitability of Kv3.3expressing, fast-spiking neurons in zebrafish. In parallel, the precision and amplitude of the startle response, a behavior that incorporates the fastest and largest-amplitude movements in the zebrafish repertoire, are significantly reduced. Decreased excitability and the locomotor phenotype are directly attributable to the dominant-negative properties of the SCA13 mutant subunit. Our results support the conclusion that changes in neuronal excitability initiate pathogenesis in SCA13 and establish zebrafish as 
an excellent model system for investigating the relationship between neuronal activity, motor control, and cell death in SCA13 and other human diseases.

\section{Materials and Methods}

Materials. The human Kv3.3 plasmid was a kind gift from Dr. James L. Rae (Mayo Foundation, Rochester, MN) (Rae and Shepard, 2000; Waters et al., 2006). The $\mathrm{R} 420 \mathrm{H}$ mutation was introduced using QuikChange (Agilent) (Waters et al., 2006).

The znp-1, zn-8, and 39.4D5 (anti-Islet) mouse monoclonal antibodies were obtained from the Developmental Studies Hybridoma Bank developed under the auspices of the National Institute of Child Health and Human Development and maintained by the University of Iowa. Goat- $\alpha$-mouse Alexa Fluor-488, goat- $\alpha$-rabbit Alexa Fluor594 , and $\alpha$-bungarotoxin $(\alpha$-BgTx) Alexa Fluor-647 were obtained from Invitrogen.

Anti-Kv3.3 antibodies were raised in rabbits by Covance Research Products against the peptide epitope CRIGADPDDPTASAHTAFK (Mock et al., 2010). The $\mathrm{N}$-terminal cysteine residue was added to couple the peptide to keyhole limpet hemocyanin. The epitope sequence (lacking the cysteine) is unique in the zebrafish genome ( $\mathrm{Zv8})$ and is located between the S5 transmembrane segment and reentrant pore loop of $\mathrm{Kv} 3.3 \mathrm{a}$, which is encoded by the $k c n c 3 a$ gene (Mock et al., 2010). In other zebrafish $k c n c$ gene products, the analogous sequence differs by $2-7$ residues. Antibody specificity was assessed on immunoblots using protein translated in vitro for wild-type $k c n c 3 a$ or $k c n c$ gene sequences that differ in the epitope by 2 or 4 aa. Only the $k c n c 3 a$ sequence was labeled, suggesting that the antibodies are specific for Kv3.3a (data not shown).

Animal maintenance and generation of transgenic zebrafish line. Animal procedures were approved by the Chancellor's Animal Research Committee at University of California at Los Angeles (UCLA). Zebrafish (Danio rerio) were housed at the UCLA Zebrafish Core Facility at $28^{\circ} \mathrm{C}$ using a $14 \mathrm{~h} / 10 \mathrm{~h}$ light/dark cycle and bred to obtain embryos.

A transgenic line of zebrafish, $\operatorname{Tg}(M m u . M N X 1-M N E$ :EGFP), that specifically expresses enhanced green fluorescent protein (EGFP) under the control of the motor neuron enhancer from the mouse MNX1 ( $\mathrm{Hb}$ ) gene was generated using a Tol2 vector (Kawakami et al., 2004). An XhoI-BamHI fragment containing the EF1p promoter upstream of the EGFP coding sequence was removed from the pT2KXIG vector and replaced by a minimal promoter from the zebrafish gata2 gene (Kawakami et al., 2004; Yang et al., 2007). The 125 bp motor neuron enhancer element was amplified from the plasmid pBlu2SKP-Hb9-ßglo-EGFP, kindly provided by Dr. Steven A. Goldman (University of Rochester, Rochester, NY) (Nakano et al., 2005). Three copies of the enhancer were inserted upstream of the gata2 promoter and the EGFP coding sequence. Plasmid DNA and in vitro transcribed RNA encoding transposase were coinjected into single-celled, wild-type (AB) zebrafish embryos, which were raised to sexual maturity and bred. Germline transgenic animals were identified in the F1 generation by EGFP expression in spinal cord motor neurons. A line expressing green fluorescent protein under the control of the zebrafish $\mathrm{Hb} 9$ motor neuron enhancer has been reported previously (Flanagan-Steet et al., 2005).

Immunocytochemistry. At 24, 48, or $72 \mathrm{hpf}$ (hours postfertilization), zebrafish embryos of either gender were fixed in $4 \%$ paraformaldehyde in PBS at $4^{\circ} \mathrm{C}$ for 3-4 h with gentle agitation (Tallafuss et al., 2009). Embryos were rinsed twice for $5 \mathrm{~min}$ in PBS at room temperature, incubated $1-2 \mathrm{~h}$ at $4^{\circ} \mathrm{C}$ in blocking buffer (5\% normal goat serum, $2 \%$ bovine serum albumin, $0.1 \%$ Triton X-100 in PBS), and then incubated overnight at $4^{\circ} \mathrm{C}$ in primary antibody diluted in PBS. Embryos were washed in PBS containing $0.1 \%$ Triton X-100 (PBST) for $1-2 \mathrm{~h}$ at room temperature and then incubated in secondary antibody diluted in blocking buffer from $2 \mathrm{~h}$ to overnight at $4^{\circ} \mathrm{C}$. Primary antibodies were used at the following dilutions: anti-Kv3.3, 1:2000; znp-1, 1:1000; zn-8, 1:400; 39.4D5, 1:100. The goat-anti-mouse Alexa Fluor-488 (green) and goat-antirabbit Alexa Fluor-594 (red) secondary antibodies were diluted 1:1000 and 1:700, respectively. Embryos were washed for $1-2 \mathrm{~h}$ in PBST, stored in $80 \%$ glycerol in PBS, and mounted in $80 \%$ glycerol with Fluoroguard (DuPont).
Fixed embryos were labeled with $\alpha$-BgTx Alexa Fluor-647 (pseudocolored green and magenta; see Fig. $2 B, C$, respectively) at $5 \mu \mathrm{g} / \mathrm{ml}$ in $1 \%$ $\mathrm{DMSO}, 5 \%$ normal goat serum in PBS for $30 \mathrm{~min}$ at room temperature, rinsed in PBS, and then labeled with primary and secondary antibodies as described above (Ono et al., 2001).

Images were acquired at the maximum resolution of $1024 \times 1024$ pixels using a Leica DM IRBE laser-scanning confocal microscope mounted with a TSC SP scanhead. The microscope was equipped with an oil-immersion, Plan Apochromat $40 \times$ objective with a numerical aperture of 1.25. Emission of the red and green fluorophores was detected using independent photomultiplier tubes. Color channels were captured sequentially and merged using Leica TCS NT software. Images show individual optical sections $(0.4884 \mu \mathrm{m})$.

Electrophysiology. RNA encoding the human Kv3.3 R420H mutant subunit $(50-100 \mathrm{pg})$ and EGFP $(20-40 \mathrm{pg})$ or EGFP alone (20-40 pg) was transcribed in vitro and injected into cells of $\mathrm{AB}$ (wild-type) zebrafish embryos at the one-cell stage. Embryos were placed in a $27.5^{\circ} \mathrm{C}$ incubator with a $14 / 10 \mathrm{~h}$ light/dark cycle using light-emitting diode strips. Animals expressing EGFP at moderate to bright intensities in their bodies were selected at $24 \mathrm{~h}$ using a Zeiss epifluorescence microscope, manually dechorionated, and returned to the incubator. Animals with dim fluorescence or EGFP expression in the yolk but not in the body were not used in our experiments.

Zebrafish embryos (2-3 days postfertilization) were anesthetized and paralyzed in recording solution containing $0.02 \%$ tricaine (MS-222) and $10 \mu \mathrm{M}$ D-tubocurarine (Sigma). Under an Olympus BX51W1 upright microscope equipped with a $40 \times$ water-immersion objective, the animals were pinned on their sides onto a Sylgard-coated Petri dish using two fine micro pins ( $1 \mathrm{~mm}$ diameter) inserted through the notochord at the belly and anus. To expose the spinal cord, the skin was removed using a glass needle. The embryos were bathed in $0.05 \%$ collagenase (type XII, Sigma) for $\sim 5 \mathrm{~min}$. The preparation was then superfused continuously $(\sim 1 \mathrm{ml} / \mathrm{min})$ with oxygenated Evan's solution containing $10 \mu \mathrm{M}$ D-tubocurarine and (in $\mathrm{mM}$ ): $134 \mathrm{NaCl}, 2.9 \mathrm{KCl}, 2.1 \mathrm{CaCl}_{2}, 1.2 \mathrm{MgCl}_{2}, 10$ HEPES, 10 glucose, adjusted to $\mathrm{pH}$ 7.8, 290 mOsm. Muscle fibers were removed using a patch pipette controlled with a micromanipulator. Muscle cells were removed by aspiration with a broken electrode.

Whole-cell patch-clamp recordings were made at room temperature $\left(22^{\circ} \mathrm{C}\right)$ at $48-72 \mathrm{hpf}$ from caudal primary $(\mathrm{CaP})$ motor neurons in RNAinjected animals (Buss and Drapeau, 2001; Buss et al., 2003). EGFPexpressing transgenic fish $[\mathrm{Tg}(\mathrm{Mmu} . M N X 1-M N E: E G F P)]$ served as uninjected controls in electrophysiological experiments. CaP neurons were identified based on their position and distinctive morphology, including a large cell body located dorsal to the ventral root and long axonal projection extending into the deep ventral musculature (Myers et al., 1986) (Fig. 1). We confirmed that patch-clamped neurons were indeed $\mathrm{CaP}$ cells by filling them with sulforhodamine $\mathrm{B}$ via the patch pipette (see Fig. $4 B$ ). The intrinsic membrane properties of CaP neurons did not differ significantly between uninjected $(n=9)$ and R420H + EGFPinjected $(n=9)$ animals. Resting membrane potentials and input resistances averaged $-62 \pm 4 \mathrm{mV}$ and $-66 \pm 5 \mathrm{mV}$, and $162 \pm 19 \mathrm{M} \Omega$ and $164 \pm 31 \mathrm{M} \Omega$ in control and $\mathrm{R} 420 \mathrm{H}$-expressing $\mathrm{CaP}$ neurons, respectively. All values are provided as mean \pm SEM. Cell body capacitance averaged $9.01 \pm 0.11 \mathrm{pF}, 9.01 \pm 0.08 \mathrm{pF}$, and $8.95 \pm 0.07 \mathrm{pF}$ in transgenic, EGFP-injected, and R420H + EGFP-injected animals, respectively. These results are in excellent agreement with the previously reported value of $8.85 \mathrm{pF}$ for CaP soma capacitance (Buss et al., 2003).

Borosilicate glass pipettes had resistances of $\sim 8 \mathrm{M} \Omega$ and were filled with the following (in $\mathrm{mm}$ ): $116 \mathrm{~K}$ gluconate, $2 \mathrm{MgCl}_{2}, 10 \mathrm{HEPES}, 10$ EGTA, $4 \mathrm{Na}_{4}$ ATP, $16 \mathrm{KCl}$, pH 7.2, $290 \mathrm{mOsm}$ ). Voltage- and currentclamp protocols were generated by a Multiclamp 700B amplifier using a Digidata 1440A interface (Molecular Devices). Data were acquired with pClamp 10.0 software, low-pass filtered at $10 \mathrm{kHz}$, and analyzed off-line with Clampfit 10.0 (Molecular Devices).

Action potential firing was evoked in current-clamp mode by injecting depolarizing currents ranging from 10 to $800 \mathrm{pA}$ in $60 \mathrm{pA}$ increments for $600 \mathrm{~ms}$. The interpulse interval was $5 \mathrm{~s}$. Average firing frequencies were measured from pulses in which current injection brought the membrane potential to $\sim-44 \mathrm{mV}$. Spike amplitude and duration were compared by 


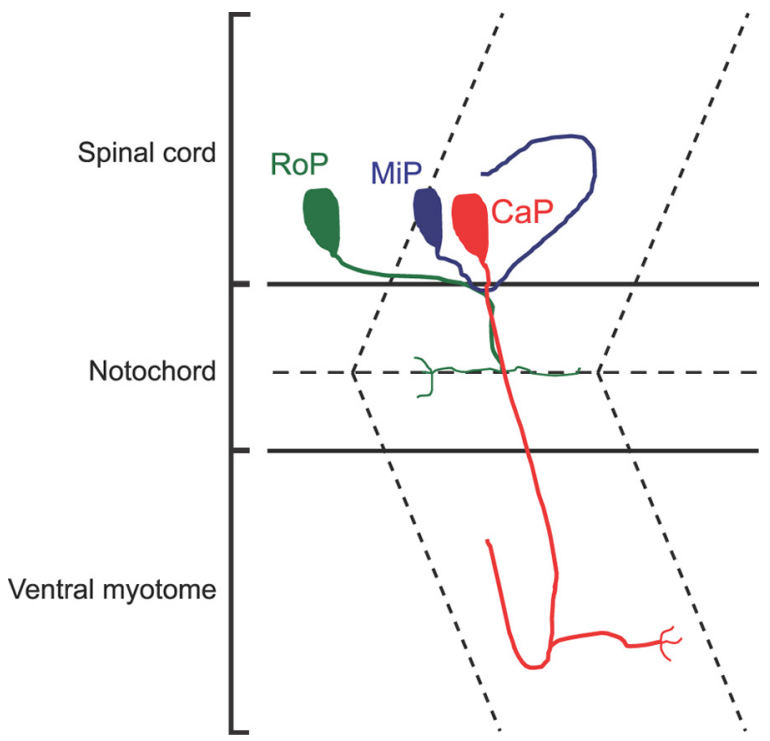

Figure 1. Schematic drawing of the morphology of the three types of spinal cord primary motor neurons, CaP (red), MiP (blue), and RoP (green) at $\sim 48$ hpf (Myers et al., 1986). Each hemisegment contains one $\mathrm{CaP}$, one MiP, and one RoP neuron. Locations of spinal cord, notochord, and ventral myotome are indicated. Dashed horizontal line is the horizontal myoseptum. Dashed chevrons mark the hemisegment boundaries.

averaging the first action potentials in trains evoked from an initial voltage of $\sim-44 \mathrm{mV}$. Mean values for the initial membrane potential after current injection were $-43.4,-44.9$, and $-43.4 \mathrm{mV}$ for uninjected, EGFP-injected, and R420H + EGFP-injected neurons, respectively. The initial voltage for each action potential was adjusted to the appropriate mean value (given above) before averaging using $0.2 \mathrm{~ms}$ windows. Averaged action potential amplitudes were measured relative to the mean initial membrane potential.

Tonic bursting activity was recorded during fictive swimming that occurred spontaneously or in response to turning on the microscope light. Episodes of fictive swimming typically lasted 1-2 min. Burst duration, interburst duration, and number of spikes per burst were measured manually using Clampfit. Values reported for the number of spikes per burst represent the averages of three consecutive burst oscillations.

Voltage-dependent ionic currents in $\mathrm{CaP}$ neurons were recorded in voltage-clamp mode. Series resistance was compensated by $\sim 80 \%$. The membrane potential was stepped from $-70 \mathrm{mV}$ to voltages ranging from -65 to $+65 \mathrm{mV}$ in $5 \mathrm{mV}$ increments, with a pulse duration of $50 \mathrm{~ms}$ and an interpulse interval of $1 \mathrm{~s}$. Linear and capacitive currents were subtracted using a $\mathrm{P} /-4$ protocol. Capacitive transients in $\mathrm{CaP}$ neurons were not well fitted by a single exponential function, indicating that inadequate space clamp may introduce systematic errors in measurements of current amplitude. It is important to emphasize that these systematic errors apply equally to CaP neurons in uninjected, EGFP-injected, and $\mathrm{R} 420 \mathrm{H}+$ EGFP-injected animals and therefore cannot account for differences in outward current amplitudes measured in these groups (see Fig. 7).

Behavioral analysis. RNA encoding R420H and EGFP or EGFP alone was injected into single-celled embryos, and animals expressing EGFP somatically were selected at $24 \mathrm{~h}$ as described above. Animals with dim fluorescence or EGFP expression in the yolk but not in the body were not assayed. AB (wild-type) zebrafish served as uninjected controls in behavioral experiments. Behavioral assays were conducted on morphologically normal animals between 55 and $60 \mathrm{hpf}$ in a room warmed to $27-28^{\circ} \mathrm{C}$. After behavioral analysis, animals were used in electrophysiological experiments as described above.

Embryos were placed in a depression slide on the stage of a Zeiss Stemi-2000C microscope. Startle responses were evoked by gentle prodding with a fine insect pin applied to the skin of the tail over the yolk sac. In zebrafish, the startle response can be elicited by a variety of sensory stimuli, including tactile, visual, and vibrational signals (Burgess and
Granato, 2007; Portugues and Engert, 2009). It is worth noting that physical contact between the probe and the animal was required to evoke the startle response in our experiments. Approach of the probe through the water did not elicit any detectable reaction. The requirement for physical contact also indicates that the behavior was not visually evoked. Therefore, tactile simulation, mediated by sensory neurons that innervate the skin, elicited the startle responses analyzed in our experiments.

Behavioral responses were captured using a high-speed digital video camera (Redlake MotionMeter or AOS X-PRI) at 1000 frames per second (fps). Each animal was assayed 2-3 times in succession at 2-3 min intervals to avoid habituation. Frames showing the animal's orientation before the response, the maximal C-start angle, and the maximal counterbend were extracted from each video. The frame before the response was used to determine the original trajectory of the animal, which was defined by a line drawn from the front of the head to the tip of the tail. The angular deviations of the head and tail from this trajectory were measured from the frame corresponding to the maximal C-start to obtain the change in head trajectory and total C-start angle (see Fig. 8D). The animal's second body bend was designated as the counterbend regardless of its angle or direction. The counterbend angle was determined by drawing a line tangential to the body at the midpoint of the bend and measuring the sum of the head and tail angles from that line (see Fig. $8 D$ ).

\section{Results}

\section{Kv3.3 channels are expressed in fast-spiking primary} motor neurons

To determine the effect of a SCA13 mutation on excitability in vivo, we first identified a population of Kv3.3-expressing neurons in zebrafish. In mammals, Kv3.3 is prominently expressed in cerebellar neurons and spinal cord motor neurons where it is localized to axons and synaptic terminals (Brooke et al., 2004; Chang et al., 2007). We investigated whether Kv3.3 is found in spinal cord neurons in zebrafish because the development of the spinal cord has been studied extensively (Lewis and Eisen, 2003). Distinct classes of neurons have been identified, each with characteristic birthdates and patterns of migration and process extension. This includes the three primary motor neurons found in each hemisegment, $\mathrm{CaP}, \mathrm{MiP}$ (middle primary), and RoP (rostral primary) (Fig. 1). The architecture of the zebrafish spinal cord is reliably reproduced from segment to segment and between different animals.

Antibodies were raised against an 18 aa peptide epitope in Kv3.3a, which is encoded by the zebrafish kcnc3a gene (Mock et al., 2010). Zebrafish embryos were stained with anti-Kv3.3 antibodies at 24,48 , and $72 \mathrm{hpf}$. Labeling was observed as early as 24 $\mathrm{hpf}$ (data not shown). At $48 \mathrm{hpf}$, a long process extending into the ventral musculature was labeled in each hemisegment (Fig. $2 A$ ). This pattern strongly resembles the distinctive morphology of CaP motor neuron axons (Fig. 1) (Myers et al., 1986). Double labeling at $48 \mathrm{hpf}$ with the anti-Kv3.3 antibody and a monoclonal antibody, znp-1, which labels the axons and nerve terminals of primary motor neurons, confirmed that Kv3.3 is expressed in the axons of CaP motor neurons (Fig. 2A) (Trevarrow et al., 1990; Fox and Sanes, 2007). Kv3.3 staining was also detected in CaP cell bodies, which were located in a different optical section from that shown in Figure $2 \mathrm{~A}$ (data not shown).

In mammals, Kv3.3 is expressed at the presynaptic side of the neuromuscular junction (Brooke et al., 2004). To determine whether Kv3.3 is expressed at synaptic sites in zebrafish, embryos were double labeled with anti-Kv3.3 antibodies and fluorescently labeled $\alpha$-BgTx (pseudo-colored green), which binds to postsynaptic acetylcholine receptors at the neuromuscular junction (Fig. $2 B$ ) (Ono et al., 2001). At $48 \mathrm{hpf}, \alpha$-BgTx and Kv3.3 labeling were juxtaposed at synaptic sites, which at this stage of development appear as en passant synaptic contacts on or near the axon shaft 
(Fig. 2 B) (Lefebvre et al., 2004). At 72 hpf, Kv3.3 staining was detected on branches extending from the $\mathrm{CaP}$ axon shaft that strongly resemble more mature motor neuron terminals (Fig. $2 B$ ).

Kv3.3 staining was also detected in the axons of MiP motor neurons, which innervate the dorsal musculature (Figs 1, $2 C)$. Kv3.3 labeling was juxtaposed with $\alpha$-BgTx staining and closely associated with znp-1 staining in MiP axons (Fig. $2 C)$. In addition, Kv3.3 staining was detected in an anterior/posterior track ventral to motor neuron cell bodies in the spinal cord (Figs. 2, 3). Some of this signal may arise from the axons of primary motor neurons, notably RoP, which course through the spinal cord to the ventral root where $\mathrm{CaP}, \mathrm{MiP}$, and RoP axons exit together into the periphery (Fig. 1). We have not investigated possible Kv3.3 expression in RoP neurons in detail, however, because at this stage of development, the peripheral RoP axon is difficult to identify unambiguously (Myers et al., 1986). We conclude that $\mathrm{Kv} 3.3$ is expressed in $\mathrm{CaP}$ and MiP (and possibly RoP) motor neurons in the zebrafish spinal cord.

To investigate whether $\mathrm{Kv} 3.3$ is expressed in the cell bodies of spinal cord sensory and secondary motor neurons, embryos were double labeled with antiKv3.3 antibodies and 39.4D5, a monoclonal antibody raised against rat Islet-1 (Fig. 3A) (Ericson et al., 1992). The Islet proteins are nuclear transcription factors expressed in motor and sensory neurons in the zebrafish spinal cord early in development (Pineda et al., 2006). Although Islet- 1 is expressed in the somas of all motor neurons, the optical section in Figure $3 A$ predominantly shows the cell bodies of secondary motor neurons, which arise later and are more numerous than primary motor neurons (Myers et al., 1986). We did not detect juxtaposition of 39.4D5 and Kv3.3 labeling in these motor neuron cell bodies (Fig. 3A). Furthermore, we did not detect Kv3.3 expression in the cell bodies of Rohon-Beard sensory neurons, which are also labeled by 39.4D5 (Fig. 3A) (Pineda et al., 2006).

To investigate whether Kv3.3 is expressed in the axons of secondary motor neurons, embryos were double labeled with anti-Kv3.3 antibodies and $\mathrm{zn}-8$, a monoclonal antibody that specifically stains the soma and fasciculated axons of secondary motor neurons (Trevarrow et al., 1990). The labeling patterns were distinct (Fig. 3B). Although the axons of $\mathrm{CaP}$ and secondary motor neurons were closely apposed, consistent with a role for
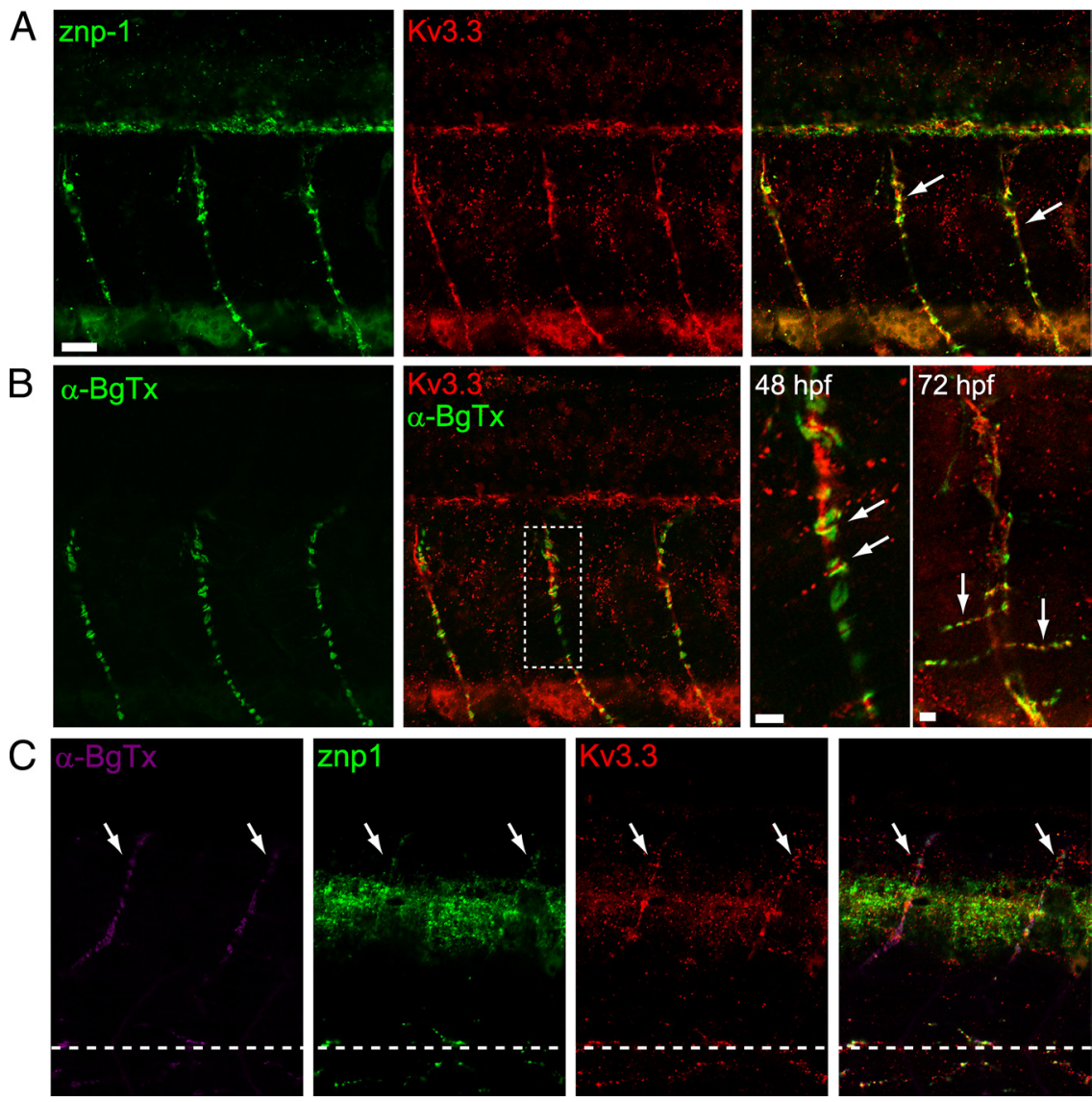

Figure 2. Kv3.3 is expressed in fast-spiking primary motor neurons. A, At $48 \mathrm{hpf}$, zebrafish embryos were labeled with znp- 1 (green), which labels the axons and terminals of primary motor neurons, and anti-Kv3.3 antibodies (red). The merged image is shown in the right panel. Arrows indicate regions of overlap. Scale bar (left image) (20 $\mu \mathrm{m}$ ) applies to all panels except where noted. In all panels, dorsal is up and anterior is to the left. $\boldsymbol{B}$, At $48 \mathrm{hpf}$, embryos were labeled with $\alpha$-BgTx coupled to Alexa Fluor-647 (pseudo-colored green), which labels postsynaptic acetylcholine receptors, and with anti-Kv3.3 antibodies (red). Images were obtained from the same animal shown $\boldsymbol{A}$. At right, the panel labeled $48 \mathrm{hpf}$ shows a close-up of the area in the dashed box in the middle panel to highlight juxtaposition of $\alpha$-BgTx and Kv3.3 labeling at synaptic sites (arrows). Scale bar, $5 \mu \mathrm{m}$, applies to the panels labeled 48 and 72 hpf. Right panel shows a 72 hpf embryo that has been labeled with znp-1 (green) and anti-Kv3.3 antibodies (red) to highlight Kv3.3 localization on side branches that resemble mature motor neuron terminals (arrows). C, Panels show labeling of MiP axons (arrows) by $\alpha$-BgTx (pseudo-colored magenta), znp-1 (green), and anti-Kv3.3 (red). Right panel shows a triple overlay. The dashed line indicates the position of the horizontal myoseptum. All images correspond to single optical sections $(0.4884 \mu \mathrm{m})$.
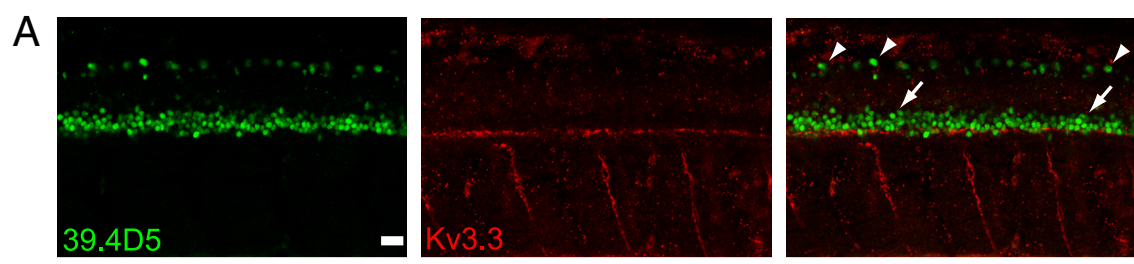

B
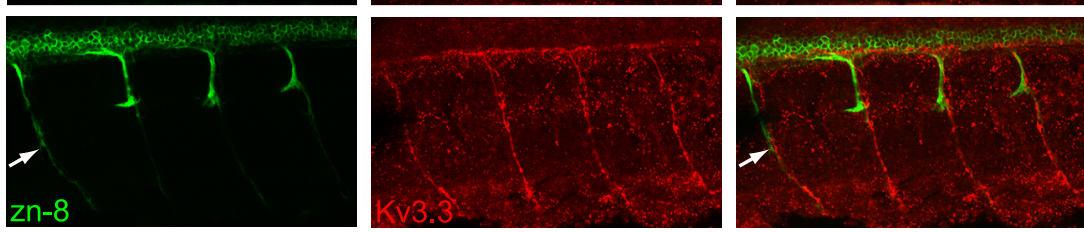

Figure 3. Kv3.3 labeling was not detected in sensory or secondary motor neurons. $\boldsymbol{A}$, At $48 \mathrm{hpf}$, embryos were labeled with 39.4D5 (green), which labels nuclei of sensory and motor neurons, and anti-Kv3.3 antibodies (red). The merged image is shown in the right panel. The arrowheads and arrows indicate the cell bodies of sensory and motor neurons, respectively. Scale bar, $20 \mu \mathrm{m}$, applies to all panels. Dorsal is up and anterior is to the left. $\boldsymbol{B}$, At $48 \mathrm{hpf}$, embryos were labeled with zn-8 (green), which labels secondary motor neurons, and anti-Kv3.3 antibodies (red). The merged image is shown in the right panel. Arrows in the left and right panels indicate the long axon of a secondary motor neuron. All images correspond to single optical sections $(0.4884 \mu \mathrm{m})$. 
A

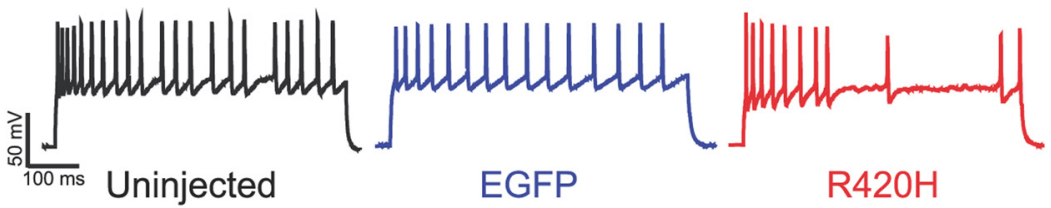

B

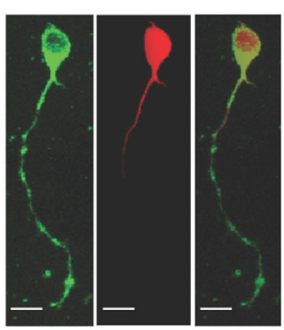

C

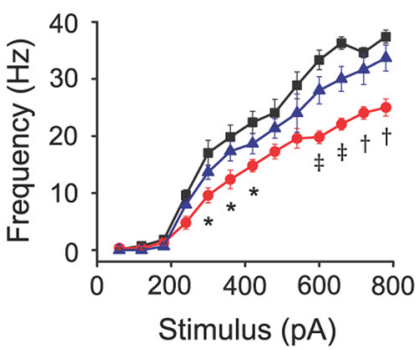

D
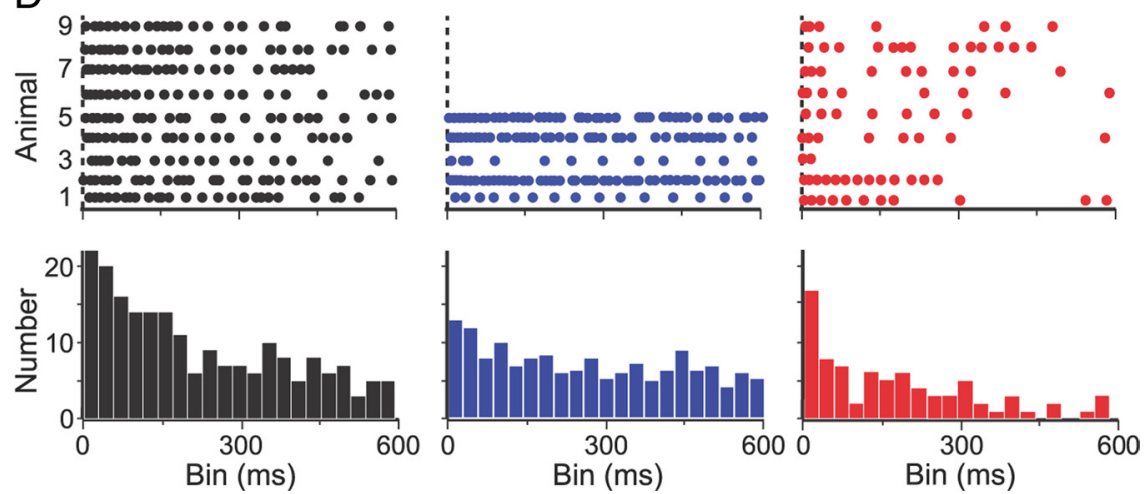

Figure 4. Expression of the $\mathrm{R} 42 \mathrm{OH}$ subunit reduces the excitability of $\mathrm{CaP}$ motor neurons. $\boldsymbol{A}$, Action potential firing was evoked in patch-clamped CaP motor neurons from uninjected (black), EGFP-injected (blue), or R420H + EGFP-injected animals (red). Spikes were evoked by depolarizing the membrane to $-25 \mathrm{mV}$. Representative results are shown. $\boldsymbol{B}$, To verify that patched cells were CaP neurons, cells were filled with sulforhodamine B via the patch pipette. A representative cell is shown. Left, EGFP fluorescence; middle, sulforhodamine B fluorescence in same neuron; right, merged image. Dorsal is up and anterior is to the left. Scale bars, $10 \mu \mathrm{m}$. C, Average action potential frequencies have been plotted versus stimulus amplitude for uninjected (black squares, $n=9$ ), EGFP-injected (blue triangles, $n=5$ ), and R420H + EGFP-injected (red circles, $n=9$ ) animals. Values are shown as mean \pm SEM, significantly different from uninjected and EGFP-injected: ${ }^{*} p<0.05,{ }^{\dagger} p<0.005,{ }^{\ddagger} p<0.0005$ by one-way ANOVA with Friedman post hoc test. $\boldsymbol{D}$, Top, Raster plots of action potentials in CaP motor neurons during evoked firing from uninjected (black), EGFP-injected (blue), or R420H + EGFP-injected (red) animals are shown. Each row represents a CaP neuron from a different animal. Bottom, Histograms show the number of evoked action potentials during 30 ms bins for uninjected (black), EGFP-injected (blue), and R420H + EGFP-injected (red) animals. In $\boldsymbol{C}$ and $\boldsymbol{D}$, spikes were evoked by depolarizing the membrane to $-20 \mathrm{mV}$.

$\mathrm{CaP}$ axons in pioneering the outgrowth of secondary motor neuron axons, labeling of $\mathrm{Kv} 3.3$ and $\mathrm{zn}-8$ was restricted to separate axonal processes and showed no significant overlap (Fig. 3B) (Myers et al., 1986).

It is important to note that failure to detect staining with the Kv3.3 antibody does not exclude the possibility that unlabeled cells express Kv3.3. Furthermore, Kv3.3 may be expressed in cell types that have not been investigated here. However, the apparent expression of Kv3.3 in primary but not secondary motor neurons is consistent with the physiological roles of these cells. In zebrafish, there are 3 primary motor neurons per segment on each side of the body (Fig. 1) (Eisen et al., 1986). They develop early, innervate fast-twitch muscle, and control rapid, large-amplitude movements including fast swimming and the startle response (Eisen et al., 1986; Liu and Westerfield, 1988; McLean et al., 2007). Primary motor neurons are fast-spiking cells that drive high-frequency muscle contractions (Buss and Drapeau, 2001; Buss et al., 2003; McLean et al., 2007). In contrast, secondary motor neurons innervate slow-twitch muscles that control slow swimming characterized by low-frequency muscle contractions (Liu and Westerfield, 1988; McLean et al., 2007).

Expression of $\mathrm{R} 420 \mathrm{H}$ specifically reduces the excitability of $\mathrm{CaP}$ motor neurons

To investigate the effect of a SCA13 mutation on neuronal excitability in vivo, we expressed the human dominant-negative $\mathrm{R} 420 \mathrm{H}$ subunit in zebrafish. The $\mathrm{R} 420 \mathrm{H}$ mutation, located in the $\mathrm{S} 4$ segment of the voltage sensor domain, causes adultonset, progressive ataxia associated with progressive degeneration of the cerebellum (Waters et al., 2006). Incorporation of $\mathrm{R} 420 \mathrm{H}$ subunits into tetrameric Kv3 channels suppresses $\mathrm{K}^{+}$current amplitude by a dominant-negative mechanism. Importantly, $\mathrm{R} 420 \mathrm{H}$ is incapable of forming active $\mathrm{K}^{+}$channels on its own and is therefore expected to be functionally silent in cells that do not express endogenous, wild-type Kv3 subunits (Waters et al., 2006). In contrast, in Kv3-expressing cells, R420H is expected to lower Kv3 current amplitude by a dominant-negative mechanism.

RNA encoding R420H and EGFP or EGFP alone was injected into the cell of single-celled, wild-type (AB) embryos. Successfully injected animals were identified at $24 \mathrm{hpf}$ by EGFP expression in their bodies. Current-clamp recordings were made in the whole-cell patch configuration at 48-72 hpf from CaP neurons in RNA-injected animals (Fig. 4A). Recordings were also made in uninjected animals using a transgenic zebrafish line $[\mathrm{Tg}(\mathrm{Mmu}$. MNX1-MNE:EGFP)] that expresses EGFP under the control of the mouse MNX1 ( $\mathrm{Hb}$ 9) motor neuron enhancer, which made it easier to identify $\mathrm{CaP}$ neurons during these control experiments $(\mathrm{Na}$ kano et al., 2005) (Fig. 4A). To confirm that the patch-clamped neurons were indeed $\mathrm{CaP}$ cells, they were filled with sulforhodamine B introduced via the patch pipette (Fig. $4 B$ ).

Action potential firing was evoked by injecting depolarizing current. Expression of the R420H subunit affected evoked firing in two ways. First, the R420H subunit significantly reduced the average firing rate compared with EGFP-injected or uninjected controls (Fig. $4 A, C$ ). In addition, $\mathrm{R} 420 \mathrm{H}$-expressing $\mathrm{CaP}$ neurons often ceased firing before the end of the $600 \mathrm{~ms}$ pulse (Fig. $4 A, D)$. In contrast, the majority of neurons from uninjected and EGFP-injected animals sustained firing throughout the pulse. The finding that $\mathrm{R} 420 \mathrm{H}$ expression decreases neuronal excitability is consistent with the special role of Kv3 channels in facilitating high-frequency, repetitive firing (see Discussion) (Rudy and McBain, 2001).

We also assessed the effect of the SCA13 mutant subunit on motor neuron activity during fictive swimming (Fig. 5). In fictive swimming, motor neurons on opposite sides of the body fire in an alternating burst pattern driven by a central pattern generator. 
In zebrafish, fictive swimming can occur spontaneously or be evoked by turning on the light (Masino and Fetcho, 2005). Bursting activity during fictive swimming was recorded in current-clamp mode from uninjected control or R420H-expressing animals (Fig. 5A). The $\mathrm{R} 420 \mathrm{H}$ subunit significantly decreased the number of spikes per burst, consistent with the reduced excitability seen during evoked firing in $\mathrm{CaP}$ motor neurons (Fig. 5B). We conclude that expression of the SCA13 mutant subunit significantly suppresses the excitability of $\mathrm{CaP}$ motor neurons during both evoked activity and fictive swimming.

In contrast, the $\mathrm{R} 420 \mathrm{H}$ subunit had no significant effects on burst duration or interburst interval during fictive swimming (Fig. 5B). The time course of the alternating firing pattern during fictive swimming is controlled by neurons of the central pattern generator rather than by the $\mathrm{CaP}$ motor neurons. This ensures that $\mathrm{CaP}$ firing on opposite sides of the body alternates in a strict fashion. Our results indicate that expression of the SCA13 mutant subunit affects the intrinsic excitability of $\mathrm{CaP}$ motor neurons but has no detectable effect on the activity of neurons that constitute the central pattern generator. Although we did not record simultaneously from $\mathrm{CaP}$ neurons on both sides of the body, we are confident that the activity of the central pattern generator evoked alternating motor neuron firing. In zebrafish, disrupting this pattern generates an accordion phenotype, characterized by a shortened body and an abnormal, scooting form of locomotion caused by simultaneous, bilateral muscle contractions (Hirata et al., 2005). In contrast, the zebrafish used in our experiments swam normally and had normal body lengths. The specific effect of the R420H subunit on $\mathrm{CaP}$ motor neurons is consistent with the finding that $\mathrm{Kv} 3.3$ expression was detected in primary motor neurons but not in other types of spinal cord neurons.

We also assessed whether the $\mathrm{R} 420 \mathrm{H}$ mutant subunit affected the properties of individual action potentials in $\mathrm{CaP}$ motor neurons. Action potentials in $\mathrm{R} 420 \mathrm{H}$-expressing neurons tended to be smaller in amplitude and longer in duration than those in cells from EGFP-injected or uninjected animals, although these trends did not reach the level of statistical significance (Fig. 6).

\section{Expression of $\mathrm{R} 420 \mathrm{H}$ decreases $\mathrm{K}^{+}$current amplitude in $\mathrm{CaP}$ motor neurons}

To verify that the $\mathrm{R} 420 \mathrm{H}$ subunit exerted a dominant-negative effect in zebrafish, we measured the amplitude of outward $\mathrm{K}^{+}$ currents in $\mathrm{CaP}$ motor neurons in voltage-clamp mode. We found that the SCA13 mutant subunit significantly reduced the amplitude of outward currents in $\mathrm{CaP}$ neurons compared with EGFP-injected or uninjected controls (Fig. $7 A, B$ ). At $+60 \mathrm{mV}$, the amplitude of the peak outward current was reduced by $\sim 34 \%$ in $\mathrm{R} 420 \mathrm{H}$-expressing cells, suggesting that Kv3 channel activity constitutes a significant fraction of the outward current in $\mathrm{CaP}$ motor neurons at this stage in development (Fig. 7B). In contrast, the $\mathrm{R} 420 \mathrm{H}$ subunit had no significant effect on the amplitude of inward currents (Fig. $7 A, C$ ). We conclude that expression of the SCA13 mutant protein specifically suppressed the amplitude of outward currents in $\mathrm{CaP}$ motor neurons, consistent with the dominant-negative effect of the $\mathrm{R} 420 \mathrm{H}$ subunit on $\mathrm{Kv} 3$ channel activity.

\section{Expression of SCA13 dominant-negative subunit reduces} precision and amplitude of the startle response

We also investigated the effect of the $\mathrm{R} 420 \mathrm{H}$ mutation on zebrafish locomotion. Animals injected with R420H and EGFP RNA or EGFP RNA alone developed normally, with no obvious delay and no gross locomotor deficits. Spontaneous coiling of the tail emerged on schedule and the animals were able to swim in response to tactile stimuli starting at $\sim 27 \mathrm{hpf}$ (Brustein et al., 2003).

We investigated the effect of the $\mathrm{R} 420 \mathrm{H}$ dominant-negative subunit on the startle response to an unexpected tactile stimulus, a behavior that is driven by the Kv3.3-expressing CaP motor neurons that innervate the deep ventral musculature (Fig. 1) (Liu and Westerfield, 1988). The startle response, which comprises the fastest and largest-amplitude movements in the zebrafish locomotor repertoire, is an early-arising behavior mediated by a simple neural circuit (Fig. 8A) (Liu and Fetcho, 1999; Korn and Faber, 2005). Tactile stimuli are detected by sensory neurons, 

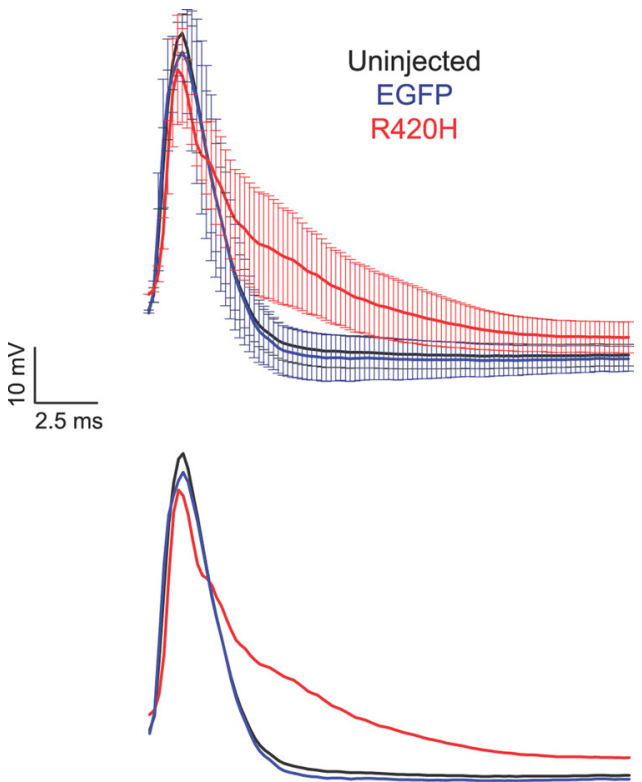

Figure 6. $\mathrm{R} 420 \mathrm{H}$ subunit increases action potential duration. The figure shows averaged action potential shapes recorded in CaP motor neurons in uninjected (black, $n=9$ ), EGFPinjected (blue, $n=5$ ), and R420H + EGFP-injected (red, $n=9$ ) animals. Mean peak amplitudes were $51.3 \pm 3.6,48.6 \pm 5.5$, and $41.8 \pm 10.1 \mathrm{mV}$ for uninjected, EGFP-injected, and $\mathrm{R} 42 \mathrm{OH}+\mathrm{EGFP}$-injected animals, respectively. Spike durations measured at half-amplitude averaged $2.5 \pm 0.31,2.5 \pm 0.35$, and $3.7 \pm 0.78 \mathrm{~s}$ for uninjected, EGFP-injected, and $\mathrm{R} 42 \mathrm{OH}+$ EGFP-injected animals, respectively. Action potentials in R420H-expressing CaP neurons tended to be smaller in amplitude and shorter in duration than those in control cells, but the measured values did not differ significantly (one-way ANOVA, $p>0.05$ ). Top, Values are shown as mean \pm SEM. Bottom, Error bars are omitted for clarity.

including trigeminal neurons that innervate the skin of the head and Rohon-Beard neurons that innervate the skin of the tail. Sensory neurons synapse onto one of two bilaterally symmetric Mauthner cells located in segment 4 and onto serial homologs in the next two segments. Upon activation, a Mauthner cell fires a single action potential that initiates the startle response. The startle response is all or none. If the Mauthner cell fires, a startle response ensues. Mauthner cell axons cross the midline to activate contralateral motor neurons, notably Kv3.3-expressing $\mathrm{CaP}$ motor neurons, as well as interneurons responsible for reciprocal inhibition of the ipsilateral motor neurons. Muscles on the contralateral side contract, generating a deep bend called the C-start, turning the animal away from the potential threat (Fig. $8 \mathrm{~B}, \mathrm{C}$ ). A subsequent counterbend is followed by rapid swimming away from the stimulus.

The startle response was assayed at 55-60 hpf in zebrafish injected with RNA encoding R420H plus EGFP or EGFP alone. Uninjected $A B$ wild-type animals were also assayed. The startle response was evoked by a gentle but unexpected prod applied to the tail above the yolk sac using a fine insect pin. The behavioral response was recorded by a high-speed digital camera at $1000 \mathrm{fps}$. Each animal was tested 2 or 3 times in succession with an interval of 2-3 min between assays to prevent habituation.

Zebrafish expressing the $\mathrm{R} 420 \mathrm{H}$ dominant-negative subunit responded to tactile stimuli with recognizable startle responses (Fig. 8C). To compare the behavior of R420H + EGFP-injected, EGFP-injected, and uninjected control animals quantitatively, we measured the change in head trajectory, the maximal C-start angle, and the maximal counterbend angle (Fig. 8D). Frames showing the animal's original trajectory, maximal C-start bend, and maximal counterbend were extracted from the video. The
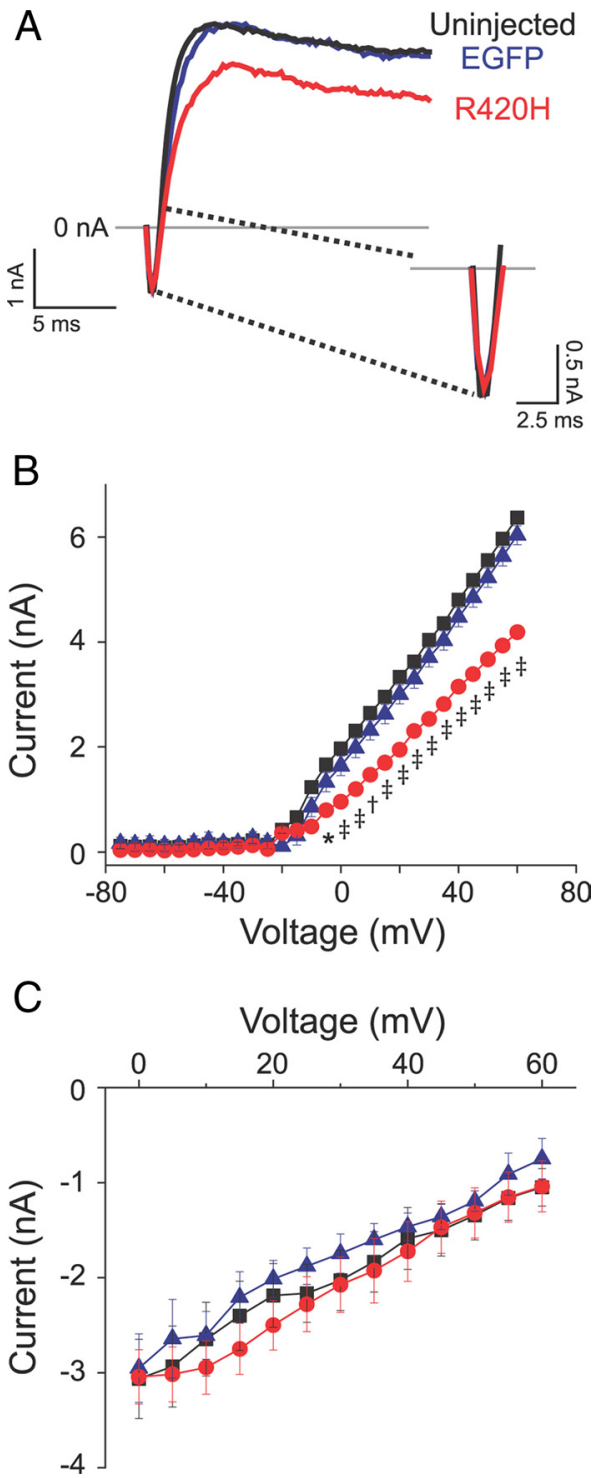

Figure 7. R420H subunit specifically reduces the amplitude of voltage-dependent outward currents in CaP motor neurons. $A$, Representative voltage-dependent currents were recorded at $+25 \mathrm{mV}$ in CaP motor neurons from uninjected (black), EGFP-injected (blue), and R420H + EGFP-injected (red) animals. Inset, Magnified view of inward currents. B, Peak outward current amplitudes have been plotted versus voltage for $\mathrm{CaP}$ motor neurons from uninjected (black squares, $n=9$ ), EGFP-injected (blue triangles, $n=5$ ), and R420H + EGFP-injected (red circles, $n=9$ ) animals. Values are shown as mean \pm SEM. If error bars are not visible, they are smaller than the symbol. ${ }^{*} p<0.05,{ }^{\dagger} p<0.005,{ }^{\ddagger} p<0.0005$, differs significantly from uninjected and EGFP-injected by one-way ANOVA with Friedman post hoc test. C, Peak inward current amplitudes have been plotted versus voltage for $\mathrm{CaP}$ motor neurons from uninjected (black squares, $n=9$ ), EGFP-injected (blue triangles, $n=5$ ), and R420H + EGFP-injected (red circles, $n=8$ ) animals. Values are shown as mean \pm SEM. If error bars are not visible, they are smaller than the symbol. Inward current amplitudes did not differ significantly.

change in head trajectory and the total C-start amplitude were determined relative to the animal's original orientation. The maximal counterbend amplitude was determined as the sum of the angles of the head and tail relative to a straight line tangent to the animal's body.

Expression of the $\mathrm{R} 420 \mathrm{H}$ subunit significantly reduced the mean change in head trajectory, C-start angle, and counterbend angle during the startle response (Fig. $9 A-C$, left). For example, in EGFP-injected zebrafish, the change in head trajectory averaged $172 \pm 5^{\circ}$, turning the animal almost directly away from the stim- 


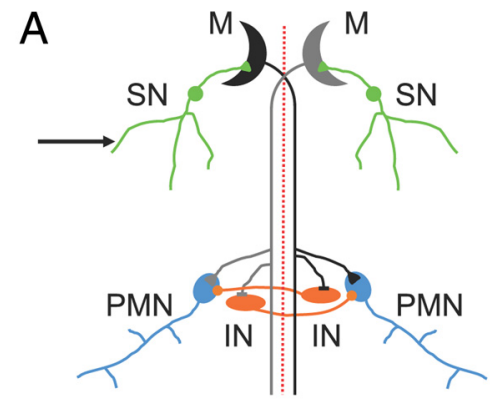

B

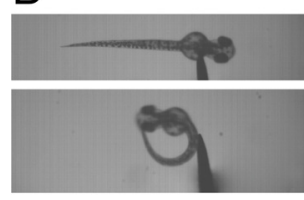

C
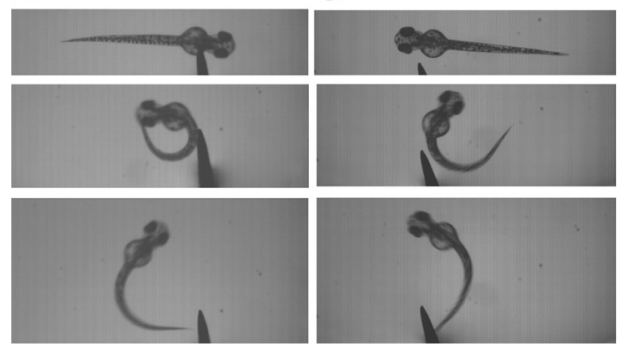

$\mathrm{D}$

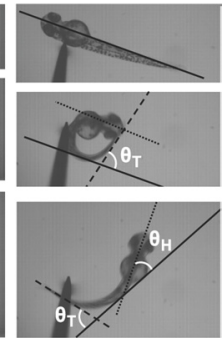

Figure 8. Startle response in zebrafish. $\boldsymbol{A}$, Simplified startle response circuitry is shown (Korn and Faber, 2005). Two bilaterally symmetric Mauthner cells (M) (black and gray) are innervated by ipsilateral sensory neurons (SN) (green). Arrow indicates tactile stimulus. Mauthner axons cross the midline to form chemical synapses on contralateral CaP motor neurons (PMN) (blue) and electrical synapses on contralateral interneurons that mediate reciprocal inhibition (IN) (orange). B, A startle response was evoked in an EGFP-injected animal at 55-60 hpf. The panels show individual frames from a high-speed video. Top, Before the stimulus. Middle, Maximal C-start. Bottom, Maximal counterbend. C, A startle response was evoked in an R420H + EGFP-injected animal at 55-60 hpf. Panels follow the description in part B. D. The startle response was characterized quantitatively as shown. Top, Solid line marks the initial orientation of the fish. Middle, The dotted and dashed lines mark the orientations of the head and tail, respectively, at the maximal C-start angle. Angular deviations of the head $\left(\theta_{H}\right)$ and tail $\left(\theta_{T}\right)$ from the original orientation (solid line) were measured. $\theta_{H}$ equals the change in head trajectory; $\theta_{\mathrm{H}}+\theta_{\mathrm{T}}$ equals the maximal C-start angle. In this example, $\theta_{\mathrm{H}}$ was close to $180^{\circ}$, so the intersection of the lines is not visible in the area shown. Bottom, The maximal counterbend angle was defined as $\theta_{\mathrm{H}}$ (dotted) $+\theta_{\mathrm{T}}$ (dashed) relative to a line tangential to the animal's body (solid). Frames in $\boldsymbol{D}$ were obtained using an uninjected animal at $55-60 \mathrm{hpf}$.

ulus. In R420H + EGFP-injected fish, the average change in head trajectory decreased significantly to $143 \pm 6^{\circ}$. The maximal C-start and counterbend angles were similarly affected (see legend to Fig. 9). In addition, the SCA13 mutant subunit increased the range of angles measured for each parameter. In contrast, the average values and range of angles measured in animals expressing EGFP alone and in uninjected animals were quite similar (Fig. 9). During the counterbend, $\mathrm{R} 420 \mathrm{H}$-expressing animals occasionally turned in the wrong direction, that is, in the same direction as the C-start, a behavior that was not observed in EGFP-injected or uninjected zebrafish in our experiments.

To compare the distributions of the measured parameters, the results obtained with $\mathrm{R} 420 \mathrm{H}+$ EGFP-injected and EGFP-injected zebrafish were binned and plotted in histogram form (Fig. 9A-C, right). The changes in head trajectory and counterbend angles were well fitted by normal distributions in R420H + EGFP- and EGFP-injected animals (Fig. 9A,C). In the R420H-expressing zebrafish, the peaks of the distributions were significantly shifted to smaller angles and the widths significantly increased compared with control animals. Although the $\mathrm{C}$-start response angles were normally distributed in EGFP-injected fish, data obtained with R420H +EGFP-injected animals were not well fitted by a Gaussian function (Fig. 9B). The binned data showed that in $\mathrm{R} 420 \mathrm{H}-$ expressing animals the $\mathrm{C}$-start angles covered a larger range, with
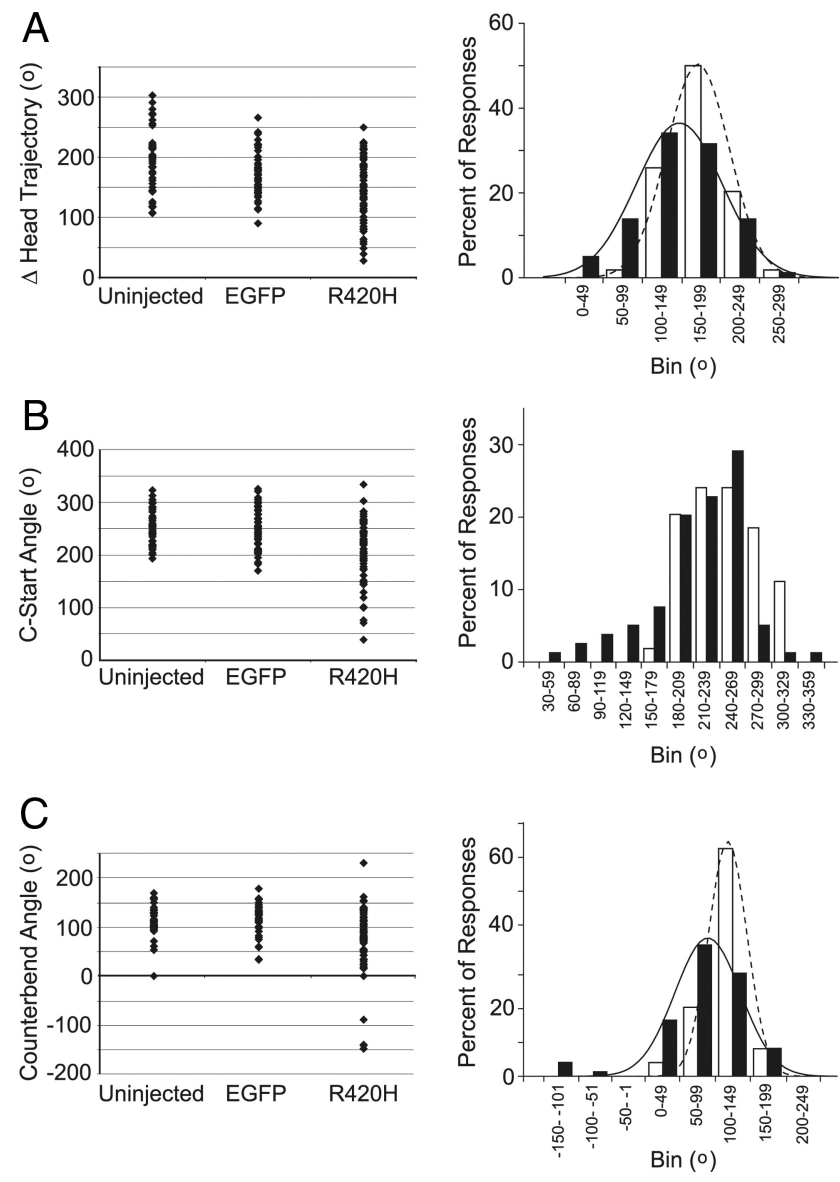

Figure 9. $\mathrm{R} 42 \mathrm{OH}$ subunit reduces the precision and amplitude of the startle response. $A-C$, Left panels show results from individual startle response assays performed on uninjected, EGFPinjected, or R420H + EGFP-injected animals: change in head trajectory $(A)$, maximum C-start angle $(\boldsymbol{B})$; maximum counterbend angle $(\boldsymbol{C}) \cdot \ln \boldsymbol{C}$, a negative counterbend angle indicates that the counterbend and the $C$-start were in the same direction. Mean change in head trajectory: uninjected, $193 \pm 8^{\circ}\left(n=43\right.$ assays, conducted on 16 animals); EGFP-injected, $172 \pm 5^{\circ}(54$ assays on 22 animals); R420H + EGFP-injected, $143 \pm 6^{\circ}$ (79 assays on 27 animals). Values are provided as mean \pm SEM. R420H differed significantly from EGFP and uninjected, $p<0.001$ by one-way ANOVA followed by Student's $t$ test. Mean maximum C-start angle: uninjected, $252 \pm$ $5^{\circ}$ (42 assays); EGFP, $245 \pm 5^{\circ}$ (54 assays); R420H + EGFP, $213 \pm 6^{\circ}$ (79 assays). Values are provided as mean \pm SEM. R420H differed significantly from EGFP and uninjected, $p<0.001$ by one-way ANOVA followed by Student's $t$ test. Mean maximum counterbend angle: uninjected, $111 \pm 5^{\circ}$ (38 assays); $\mathrm{EGFP}, 115 \pm 4^{\circ}$ (49 assays); R420H, $79 \pm 8^{\circ}$ ( 72 assays). R420H differed significantly from EGFP and uninjected, $p<0.01$ by one-way ANOVA followed by Student's $t$ test. There were fewer counterbend measurements because the head or the tail was sometimes out of frame during this phase of the response. In the right panels, results from startle response assays performed on EGFP-injected (open bars) or R420 + EGFP-injected (black bars) animals have been binned and plotted versus percentage of total responses: change in head trajectory $(\boldsymbol{A})$, maximum $\boldsymbol{C}$-start angle $(\boldsymbol{B})$, maximum counterbend angle $(\boldsymbol{C})$. In $\boldsymbol{A}$ and $\boldsymbol{C}$, binned data have been fitted with Gaussian functions of the form $y=\left(A /\left(w^{*} \sqrt{(\pi / 2)}\right)\right)^{*} \exp \left(-2^{*}\left(\left(x-x_{c} / w\right) \wedge 2\right)\right.$, where $A$ is area, $w$ is width at half-maximal amplitude, and $x_{c}$ is center of the distribution. The fits are superimposed on the histograms: EGFP-injected, dashed line; R42OH + EGFP-injected, solid line. The fitted distributions of change in head trajectory $(\boldsymbol{A})$ and maximum counterbend angle $(\boldsymbol{C})$ for EGFP- and $\mathrm{R} 420 \mathrm{H}+\mathrm{EGFP}$-injected animals differed significantly, $p<0.01$ using $F$ test in Origin 7. Fitted parameters, change in head trajectory: EGFP, $A=100.6 \pm 0.8, w=1.59 \pm 0.01, x_{\mathrm{c}}=4.9 \pm 0.01$; $\mathrm{R} 420 \mathrm{H}, A=99.9 \pm 2.8, w=2.18 \pm 0.07, x_{\mathrm{c}}=4.4 \pm 0.04$. Fitted parameters, maximum counterbend angle: EGFP, $A=96.1 \pm 2.8, w=1.1 \pm 0.04, x_{\mathrm{c}}=5.9 \pm 0.03 ; \mathrm{R} 420 \mathrm{H}, A=96.5 \pm 4.5$, $w=1.9 \pm 0.1, x_{\mathrm{c}}=5.3 \pm 0.05$. Data in $\boldsymbol{B}$, right panel, were not fitted because the $\mathrm{R} 420 \mathrm{H}$ results were not well described by a normal distribution.

prominent skewing to smaller responses compared with EGFPinjected controls. The smaller average values and increased range of response angles measured for the three parameters indicate that expression of the $\mathrm{R} 420 \mathrm{H}$ subunit significantly reduced the 
strength and reproducibility of muscle contractions during the startle response.

\section{Discussion \\ SCA13 mutation reduces neuronal excitability and impairs locomotor behavior}

Our results provide the first demonstration that SCA13 mutations alter excitability in vivo, strongly supporting the idea that changes in neuronal activity initiate pathogenesis in SCA13 in humans. We have shown that expressing a dominant-negative SCA13 mutant subunit decreases the excitability of Kv3.3expressing, fast-spiking neurons in zebrafish. During evoked activity, $\mathrm{CaP}$ neurons expressing the $\mathrm{R} 420 \mathrm{H}$ subunit fired at a lower average frequency and exhibited a reduced capacity for sustained firing compared with control cells. During fictive swimming, the $\mathrm{R} 420 \mathrm{H}$ subunit decreased the number of spikes per burst. The effects of the mutant subunit on $\mathrm{CaP}$ excitability were specific because burst properties controlled by neurons of the central pattern generator were unaffected.

Primary motor neurons, particularly $\mathrm{CaP}$, which sends its axons deep into the ventral musculature, drive the rapid, largeamplitude muscle contractions that underlie the startle response (Liu and Westerfield, 1988; Liu and Fetcho, 1999; McLean et al., 2007; McLean and Fetcho, 2009). Expression of the R420H subunit impaired execution of the startle response to tactile stimuli, reducing the amplitude and increasing the variability of muscle contractions that generate the C-start and subsequent counterbend. These changes in motor behavior are reminiscent of gait changes in human ataxia, which is characterized by decreased step length and increased variability of step size (Palliyath et al., 1998). Thus, ataxia in humans and expression of the dominantnegative $\mathrm{R} 420 \mathrm{H}$ subunit in zebrafish result in a decrease in the precision and amplitude of sequential steps in a motor program.

The all-or-none startle response is initiated when one of the Mauthner cells fires a single action potential (Liu and Fetcho, 1999; Korn and Faber, 2005). Therefore, the reduced precision and amplitude of the startle response in $\mathrm{R} 420 \mathrm{H}$-expressing zebrafish result from changes in circuit function that occur after the Mauthner cell fires. Given the simplicity of the circuit underlying the startle response and the essential role of $\mathrm{CaP}$ neurons in driving the behavior, our results strongly suggest that reduced $\mathrm{CaP}$ excitability, including decreases in sustained firing, is a significant factor underlying the weaker and more variable muscle contractions observed during the startle response in $\mathrm{R} 420 \mathrm{H}$-expressing animals (McLean and Fetcho, 2009). This does not preclude the possibility that changes in the activity of other Kv3-expressing cells also contribute to the locomotor phenotype.

\section{Decrease in CaP excitability reflects the role of Kv3 channels in facilitating sustained, high-frequency firing in neurons}

Our data strongly support the conclusion that reduced $\mathrm{CaP}$ excitability and the locomotor phenotype were directly caused by the subfamily-specific dominant-negative effect of R $420 \mathrm{H}$ on Kv3 current amplitude (Waters et al., 2006). The R420H subunit significantly decreased the amplitude of outward currents in CaP neurons, but had no effect on the amplitude of inward currents. Importantly, the decrease in $\mathrm{K}^{+}$current amplitude was correlated with reduced excitability in $\mathrm{CaP}$ neurons. This seemingly paradoxical result is consistent with the known physiological role of Kv3 channels (Rudy and McBain, 2001). Whereas reducing the amplitude of most $\mathrm{K}^{+}$currents would be expected to increase neuronal excitability, this is not the case for Kv3 channels, which have a low open probability at the resting potential and at threshold (Rudy and McBain, 2001). Rather, Kv3 channels open primarily during action potentials to promote fast spike repolarization and rapid recovery of voltage-gated $\mathrm{Na}^{+}$channels from inactivation. The $\mathrm{R} 420 \mathrm{H}$ subunit decreased the average firing frequency, the capacity for sustained firing and action potential amplitude during evoked firing, consistent with the accumulation of $\mathrm{Na}^{+}$channel inactivation. In addition, the SCA13 mutation tended to increase action potential duration, reflecting the role of Kv3 current in spike repolarization.

\section{Zebrafish is an excellent model system for investigating pathogenic mechanisms in SCA13}

The zebrafish is a vertebrate model system with significant advantages for investigating neuronal development and great potential for analyzing nervous system function and the neural control of behavior (Brustein et al., 2003; McLean et al., 2007; Fetcho et al., 2008; Moreno and Ribera, 2009). The zebrafish is attractive as a model system for SCA13 for several reasons. First, locomotion is under cerebellar control in both zebrafish and higher vertebrates (Volkmann et al., 2008; Bae et al., 2009). Second, a variety of motor behaviors, including the startle response, evoked and spontaneous swimming, the vestibuloocular reflex, the optokinetic reflex, and the optomotor response, develop during the first week postfertilization (Baier, 2000; Brustein et al., 2003). These behaviors are highly reproducible and amenable to quantitative analysis. Third, neurons that control a variety of locomotor behaviors are accessible for electrophysiological analysis (McLean et al., 2007; Moreno and Ribera, 2009). Furthermore, the activity of zebrafish neurons can be recorded optically in living animals (McLean and Fetcho, 2008). We have shown that the novel gating properties of mammalian $\mathrm{Kv} 3$ channels are conserved in zebrafish Kv3.3, consistent with its role in facilitating fast, repetitive firing (Mock et al., 2010). SCA13 mutations have similar functional effects in zebrafish and human Kv3.3 channels (Mock et al., 2010).

Although ataxia in SCA13 is mainly cerebellar in origin, in this initial study we chose to investigate the effects of a SCA13 mutant subunit on the startle response, a behavior that does not require cerebellar input, for the following reasons. First, we have established that Kv3.3 is expressed in the fast-spiking CaP neurons that innervate the deep ventral musculature and thereby drive the startle response (Liu and Westerfield, 1988; Liu and Fetcho, 1999; McLean et al., 2007; McLean and Fetcho, 2009). Second, the behavior is well characterized, amenable to quantitative analysis, and controlled by a simple circuit, making it feasible to identify neurons whose excitability is affected by $\mathrm{R} 420 \mathrm{H}$ (Korn and Faber, 2005). Third, as in humans, the startle response emerges early in zebrafish development, within the window in which injected RNA is expected to be actively transcribed (Burgess and Granato, 2007; McLean and Fetcho, 2009). The RNA expression strategy takes advantage of the fact that $\mathrm{R} 420 \mathrm{H}$ does not function in cells that do not normally express Kv3 channels. In addition, the expression level can be controlled by varying the amount of RNA injected. As a result, we were able to obtain a mild phenotype, making it feasible to determine whether the SCA13 mutation caused quantitative differences in a well characterized behavior. Our R420H-expressing animals developed normally and were able to carry out genuine startle responses, identified by the rapidity of the initial muscle contraction (the C-start) (data not shown) and the fact that a significant fraction of responses in $\mathrm{R} 420 \mathrm{H}$ animals were within the range seen in control animals (Fig. 9). 
To investigate the role of Kv3.3 activity in excitability and locomotor control in zebrafish, we chose the dominant-negative approach rather than morpholino-mediated knockdown of Kv3.3 expression because SCA13 is not caused by a simple reduction in Kv3.3 function (Waters et al., 2006). A dominant-negative subunit will reduce the expression not just of Kv3.3 but also of any other Kv3 subunit expressed in the same cell. This may be particularly important for the etiology of SCA13 because mammalian neurons tend to express more than one type of Kv3 subunit. For instance, Purkinje cells express a combination of Kv3.3 and $\mathrm{Kv} 3.4$, whereas cerebellar granule cells express $\mathrm{Kv} 3.3$ and Kv3.1 (Weiser et al., 1994; Martina et al., 2007). Significantly, knocking out Kv3.3 in mice generates at most a subtle locomotor phenotype, whereas knocking out Kv3.3 and Kv3.1 simultaneously is much more severe, consistent with the idea that the dominant nature of the SCA13 mutations is an important factor in the development of the disease (Matsukawa et al., 2003).

In this study, we have shown that expression of a SCA13 mutation alters the functional activity of fast-spiking, Kv3.3expressing neurons in vivo and impairs locomotor behavior. Changes in excitability and behavior are directly attributable to the dominant-negative effect of the SCA13 mutant subunit. Our data provide strong evidence that changes in neuronal excitability initiate pathogenesis in SCA13. To identify cellular mechanisms responsible for SCA13, it is essential to investigate the relationship between altered neuronal function, locomotor deficits, and changes in neuronal viability. Our results establish zebrafish as an excellent vertebrate model system for investigating pathogenic mechanisms that may contribute to the etiology of SCA13 and other human diseases that affect the structure and function of the nervous system (Flinn et al., 2008; Carlson et al., 2009; Kabashi et al., 2010).

\section{References}

Bae YK, Kani S, Shimizu T, Tanabe K, Nojima H, Kimura Y, Higashijima S, Hibi M (2009) Anatomy of zebrafish cerebellum and screen for mutations affecting its development. Dev Biol 330:406-426.

Baier H (2000) Zebrafish on the move: towards a behavior-genetic analysis of vertebrate vision. Curr Opin Neurobiol 10:451-455.

Brooke RE, Moores TS, Morris NP, Parson SH, Deuchars J (2004) Kv3 voltage-gated potassium channels regulate neurotransmitter release from mouse motor nerve terminals. Eur J Neurosci 20:3313-3321.

Brustein E, Saint-Amant L, Buss RR, Chong M, McDearmid JR, Drapeau P (2003) Steps during the development of the zebrafish locomotor network. J Physiol Paris 97:77-86.

Burgess HA, Granato M (2007) Sensorimotor gating in larval zebrafish. J Neurosci 27:4984-4994.

Buss RR, Drapeau P (2001) Synaptic drive to motoneurons during fictive swimming in the developing zebrafish. J Neurophysiol 86:197-210.

Buss RR, Bourque CW, Drapeau P (2003) Membrane properties related to the firing behavior of zebrafish motoneurons. J Neurophysiol 89:657-664.

Carlson KM, Melcher L, Lai S, Zoghbi HY, Clark HB, Orr HT (2009) Characterization of the zebrafish atxh1/axh gene family. J Neurogenet 23:313-323.

Chang SY, Zagha E, Kwon ES, Ozaita A, Bobik M, Martone ME, Ellisman MH, Heintz N, Rudy B (2007) Distribution of Kv3.3 potassium channel subunits in distinct neuronal populations of mouse brain. J Comp Neurol 502:953-972.

Eisen JS, Myers PZ, Westerfield M (1986) Pathway selection by growth cones of identified motoneurones in live zebra fish embryos. Nature 320:269-271.

Ericson J, Thor S, Edlund T, Jessell TM, Yamada T (1992) Early stages of motor neuron differentiation revealed by expression of homeobox gene Islet-1. Science 256:1555-1560.

Fetcho JR, Higashijima S, McLean DL (2008) Zebrafish and motor control over the last decade. Brain Res Rev 57:86-93.
Figueroa KP, Minassian NA, Stevanin G, Waters M, Garibyan V, Forlani S, Strzelczyk A, Bürk K, Brice A, Dürr A, Papazian DM, Pulst SM (2010) KCNC3: phenotype, mutations, channel biophysics—a study of $260 \mathrm{fa}-$ milial ataxia patients. Hum Mutat 31:191-196.

Flanagan-Steet H, Fox MA, Meyer D, Sanes JR (2005) Neuromuscular synapses can form in vivo by incorporation of initially aneural postsynaptic specializations. Development 132:4471-4481.

Flinn L, Bretaud S, Lo C, Ingham PW, Bandmann O (2008) Zebrafish as a new animal model for movement disorders. J Neurochem 106:19911997.

Fox MA, Sanes JR (2007) Synaptotagmin I and II are present in distinct subsets of central synapses. J Comp Neurol 503:280-296.

Hirata H, Saint-Amant L, Downes GB, Cui WW, Zhou W, Granato M, Kuwada JY (2005) Zebrafish bandoneon mutants display behavioral defects due to a mutation in the glycine receptor $\beta$-subunit. Proc Natl Acad Sci U S A 102:8345-8350.

Kabashi E, Champagne N, Brustein E, Drapeau P (2010) In the swim of things: recent insights to neurogenetic disorders from zebrafish. Trends Genet 26:373-381.

Kawakami K, Takeda H, Kawakami N, Kobayashi M, Matsuda N, Mishina M (2004) A transposon-mediated gene trap approach identifies developmentally regulated genes in zebrafish. Dev Cell 7:133-144.

Korn H, Faber DS (2005) The Mauthner cell half a century later: a neurobiological model for decision making? Neuron 47:13-28.

Lefebvre JL, Ono F, Puglielli C, Seidner G, Franzini-Armstrong C, Brehm P, Granato M (2004) Increased neuromuscular activity causes axonal defects and muscular degeneration. Development 131:2605-2618.

Lewis KE, Eisen JS (2003) From cells to circuits: development of the zebrafish spinal cord. Prog Neurobiol 69:419-449.

Liu DW, Westerfield M (1988) Function of identified motoneurones and coordination of primary and secondary motor systems during zebrafish swimming. J Physiol 403:73-89.

Liu KS, Fetcho JR (1999) Laser ablations reveal functional relationships of segmental hindbrain neurons in zebrafish. Neuron 23:325-335.

Martina M, Metz AE, Bean BP (2007) Voltage-dependent potassium currents during fast spikes of rat cerebellar Purkinje neurons: Inhibition by BDS-1 toxin. J Neurophysiol 97:563-571.

Masino MA, Fetcho JR (2005) Fictive swimming motor patterns in wild type and mutant larval zebrafish. J Neurophysiol 93:3177-3188.

Matsukawa H, Wolf AM, Matsushita S, Joho RH, Knöpfel T (2003) Motor dysfunction and altered synaptic transmission at the parallel fiberPurkinje cell synapse in mice lacking potassium channels Kv3.1 and Kv3.3. J Neurosci 23:7677-7684.

McLean DL, Fetcho JR (2008) Using imaging and genetics in zebrafish to study developing spinal circuits in vivo. Dev Neurobiol 68:817-834.

McLean DL, Fetcho JR (2009) Spinal interneurons differentiate sequentially from those driving the fastest swimming movements in larval zebrafish to those driving the slowest. J Neurosci 29:13566-13577.

McLean DL, Fan J, Higashijima S, Hale ME, Fetcho JR (2007) A topographic map of recruitment in spinal cord. Nature 446:71-75.

Mock AF, Richardson JL, Hsieh JY, Rinetti G, Papazian DM (2010) Functional effects of spinocerebellar ataxia type 13 mutations are conserved in zebrafish Kv3.3 channels. BMC Neurosci 11:99.

Moreno RL, Ribera AB (2009) Zebrafish motor neuron subtypes differ electrically prior to axonal outgrowth. J Neurophysiol 102:2477-2484.

Myers PZ, Eisen JS, Westerfield M (1986) Development and axonal outgrowth of identified motoneurons in the zebrafish. J Neurosci 6:2278-2289.

Nakano T, Windrem M, Zappavigna V, Goldman SA (2005) Identification of a conserved 125 base-pair $\mathrm{Hb} 9$ enhancer that specifies gene expression to spinal motor neurons. Dev Biol 283:474-485.

Nelson R (2006) Potassium channels have a key role in neurodegeneration. Lancet Neurol 5:298-299.

Ono F, Higashijima S, Shcherbatko A, Fetcho JR, Brehm P (2001) Paralytic zebrafish lacking acetylcholine receptors fail to localize rapsyn clusters to synapse. J Neurosci 21:5439-5448.

Palliyath S, Hallett M, Thomas SL, Lebiedowska MK (1998) Gait in patients with cerebellar ataxia. Mov Disord 13:958-964.

Paulson HL (2009) The spinocerebellar ataxias. J Neuroophthalmol 29:227-237.

Pineda RH, Svoboda KR, Wright MA, Taylor AD, Novak AE, Gamse JT, Eisen 
JS, Ribera $\mathrm{AB}$ (2006) Knockdown of $\mathrm{Na}_{\mathrm{v}} 1.6 \mathrm{Na}^{+}$channels affects zebrafish motoneuron development. Development 133:3827-3836.

Portugues R, Engert F (2009) The neural basis of visual behaviors in the larval zebrafish. Curr Opin Neurobiol 19:644-647.

Rae JL, Shepard AR (2000) Kv3.3 potassium channels in lens epithelium and corneal endothelium. Exp Eye Res 70:339-348.

Rudy B, McBain CJ (2001) Kv3 channels: Voltage-gated $\mathrm{K}^{+}$channels designed for high-frequency repetitive firing. Trends Neurosci 24:517-526.

Supnet C, Bezprozvanny I (2010) The dysregulation of intracellular calcium in Alzheimer disease. Cell Calcium 47:183-189.

Tallafuss A, Trepman A, Eisen JS (2009) DeltaA mRNA and protein distribution in the zebrafish nervous system. Dev Dyn 238:3226-3236.

Trevarrow B, Marks DL, Kimmel CB (1990) Organization of the hindbrain segments in the zebrafish embryo. Neuron 4:669-679.
Volkmann K, Rieger S, Babaryka A, Köster RW (2008) The zebrafish cerebellar rhombic lip is spatially patterned in producing granule cell populations of different functional compartments. Dev Biol 313:167-180.

Waters MF, Minassian NA, Stevanin G, Figueroa KP, Bannister JP, Nolte D, Mock AF, Evidente VG, Fee DB, Müller U, Dürr A, Brice A, Papazian DM, Pulst SM (2006) Mutations in the voltage-gated potassium channel KCNC3 cause degenerative and developmental CNS phenotypes. Nat Genet 38:447-451.

Weiser M, Vega-Saenz de Miera E, Kentros C, Moreno H, Franzen L, Hillman D, Baker H, Rudy B (1994) Differential expression of Shaw-related $\mathrm{K}^{+}$ channels in the rat central nervous system. J Neurosci 14:949-972.

Yang Z, Jiang H, Zhao F, Shankar DB, Sakamoto KM, Zhang MQ, Lin S (2007) A highly conserved regulatory element controls hematopoietic expression of GATA-2 in zebrafish. BMC Dev Biol 7:97. 